\title{
Identification d'un deuxième gène de la maladie de Fanconi
}

La maladie de Fanconi, Fanconi anemia pour les Anglo-Saxons, est une affection autosomique récessive rare associant un syndrome malformatif variable (anomalies de phalanges du pouce, faciès particulier, microcéphalie, malformations urogénitales, etc.) à une aplasie médullaire. Cette dernière survient curieusement de façon retardée, mais inéluctable, le plus souvent entre la cinquième et la dixième année de vie. Le seul traitement curatif actuel de l'aplasie est l'allogreffe de moelle. Il existe aussi une susceptibilité aux cancers, en particulier aux leucémies aiguës. Des anomalies caryotypiques multiples sont décelées dans les lymphocytes de ces patients, accentuées par l'exposition in vitro aux agents alkylants, ce qui a pu faire considérer cette maladie comme une maladie de la réparation de l'ADN. Cette sensibilité spécifique aux agents pontants et la détection d'anomalies du cycle cellulaire sont actuellement à la base du diagnostic biologique de la maladie de Fanconi [1]. Cinq groupes de complémentation (A à $\mathrm{E}$ ) de fréquence variable ont été individualisés. $F A C$, le gène du groupe $\mathrm{C}(10-$ $15 \%$ des cas), a été localisé sur la bande chromosomique 9q22.3 puis cloné en 1992 [2]. Deux autres gènes ont été plus récemment localisés, $F A D$ en 3p22-26 et FAA en $16 \mathrm{q} 24.3$ $\left(\mathrm{m} / \mathrm{s} n^{\circ} 2\right.$, vol. 12, p. 258). C'est ce dernier gène, $F A A$, présumé en cause dans près de deux tiers des cas de maladie de Fanconi, qui vient d'être identifié simultanément par un ensemble de chercheurs hollandais et canadiens [3] et par le consortium international de l'anémie de Fanconi [4]. Les premiers ont cloné un ADNc directe des cellules des patients du groupe A, suivant la stratégie employée avec succès pour l'identification de FAC. Les seconds ont procédé à un classique clonage positionnel à partir d'une banque de cosmides. Un transcrit FAA majoritaire d'environ 5 $\mathrm{kb}$ est détecté dans de nombreux tissus, ce qui est en accord avec la notion d'un syndrome polymalformatif. La séquence de l'ADNc permet de prédire une grande protéine de 1455 acides aminés, riche en hélices $\alpha$. L'analyse de la séquence des protéines FAA et FAC ne révèle pas d'analogie avec les protéines connues des banques de données. Il s'agit donc de protéines orphelines, qui n'ont pas non plus d'analogie entre elles. Alors que FAC est une protéine cytoplasmique, l'analyse de la séquence de FAA montre deux courts motifs chevauchants susceptibles d'être des signaux de localisation nucléaire. L'ensemble de ces données suggère l'existence d'une voie biochimique nouvelle et originale, impliquée dans la protection de l'ADN, et dans laquelle chacune des protéines FA aurait une fonction [5]. Les analyses préliminaires du gène FAA chez quelques patients ont révélé la présence de délétions intragéniques, avec apparemment un effet fondateur dans plusieurs zones géographiques. Ces anomalies décalent la phase de lecture et aboutissent à des protéines plus ou moins largement tronquées. Il est très vraisemblable que l'identification des gènes $F A$ et la description des mutations ou délétions en cause auront des conséquences pour la prise en charge des patients et de leur famille. Le diagnostic biologique de la maladie sera évidemment plus précis s'il devient un criblage d'allèles mutés ou une détection de protéine tronquée. Cela permettra également d'identifier les porteurs hétérozygotes et de poser une indication de diagnostic prénatal. On peut en outre prévoir, par analogie avec $F A C$, que l'inventaire des mutations de $F A A$ révélera une corrélation entre le génotype et l'intensité du phénotype, notamment en ce qui concerne l'âge de survenue et la gravité de l'aplasie médullaire, ce qui pourrait être exploité pour optimiser la date de la greffe de moelle. Enfin, l'identification des gènes $F A$ permet d'envisager des procédures de thérapie génique. Il n'est en effet pas interdit d'espérer que les cellules souches hématopoiétiques FA corrigées par le gène déficient présenteront in vivo le même avantage de survie que celui observé in vitro. Dans cet esprit certaines équipes collectent les cellules souches des jeunes patients avant le début de l'aplasie et les conservent en vue d'une thérapie génique potentielle.

J.S.

D.L.

1. Moustachi E. Biologie cellulaire et moléculaire de l'anémie de Fanconi. Med Sci 1994 ; 10 : 97985 .

2. Strahdee CA, Gavish H, Shannon WR, Buchwald M. Cloning of Fanconi anaemia cDNAs through functional complementation. Nature 1992 ; 356 : 763-7.

3. Lo Ten Foe JR, Roojmans MA, Bosnovan-Collins L, Alon N, et al. Expression cloning of a cDNA for the major Fanconi anaemia gene, FAA. Nature Genet 1996; $14: 320-3$.

4. The Fanconi anaemia/Breast cancer consortium. Positional cloning of the Fanconi anaemia group A gene. Nature Genet $1996 ; 14$ : 324-8.

5 . D'Andrea AD. Fanconi anaemia forges a novel pathway. Nature Genet 1996 ; 14 : 240-2. 